\title{
TRPV4 Inhibition Exerts Protective Effects Against Resistive Breathing Induced Lung Injury
}

\author{
Dimitrios Toumpanakis', Athanasia Chatzianastasiou', Vyronia Vassilakopoulou (D)', Eleftheria Mizi', \\ Maria Dettoraki', Fotis Perlikos (1D', Georgia Giatra ${ }^{1,2}$, Nikolaos Mikos ${ }^{3}$, Stamatios Theocharis ${ }^{4}$, \\ Theodoros Vassilakopoulos ${ }^{1,2}$
}

I"Marianthi Simou" Applied Biomedical Research and Training Center, Evangelismos Hospital, Medical School, National and Kapodistrian University of Athens, Athens, Greece; ${ }^{2} 3^{\text {rd }}$ Department of Critical Care Medicine, Evgenideio Hospital, Medical School, National and Kapodistrian University of Athens, Athens, Greece; ${ }^{3}$ Allergology Department, Laiko General Hospital, Athens, Greece; ${ }^{4}$ First Department of Pathology, Medical School, National and Kapodistrian University of Athens, Athens, Greece

Correspondence: Dimitrios Toumpanakis, Email dtoumpanakis@yahoo.gr

Introduction: TRPV4 channels are calcium channels, activated by mechanical stress, that have been implicated in the pathogenesis of pulmonary inflammation. During resistive breathing (RB), increased mechanical stress is imposed on the lung, inducing lung injury. The role of TRPV4 channels in RB-induced lung injury is unknown.

Materials and Methods: Spontaneously breathing adult male C57BL/6 mice were subjected to RB by tracheal banding. Following anaesthesia, mice were placed under a surgical microscope, the surface area of the trachea was measured and a nylon band was sutured around the trachea to reduce area to half. The specific TRPV4 inhibitor, HC-067047 (10 mg/kg ip), was administered either prior to RB and at $12 \mathrm{hrs}$ following initiation of RB (preventive) or only at $12 \mathrm{hrs}$ after the initiation of RB (therapeutic protocol). Lung injury was assessed at $24 \mathrm{hrs}$ of RB, by measuring lung mechanics, total protein, BAL total and differential cell count, KC and IL-6 levels in BAL fluid, surfactant Protein (Sp)D in plasma and a lung injury score by histology.

Results: RB decreased static compliance (Cst), increased total protein in BAL ( $p<0.001)$, total cell count due to increased number of both macrophages and neutrophils, increased KC and IL-6 in BAL ( $p<0.001$ and $p=0.01$, respectively) and plasma SpD ( $p<0.0001)$. Increased lung injury score was detected. Both preventive and therapeutic HC-067047 administration restored Cst and inhibited the increase in total protein, KC and IL-6 levels in BAL fluid, compared to RB. Preventive TRPV4 inhibition ameliorated the increase in BAL cellularity, while therapeutic TRPV4 inhibition exerted a partial effect. TRPV4 inhibition blunted the increase in plasma $\mathrm{SpD}(\mathrm{p}<0.001)$ after RB and the increase in lung injury score was also inhibited.

Conclusion: TRPV4 inhibition exerts protective effects against RB-induced lung injury.

Keywords: resistive breathing, TRPV4, lung permeability, pulmonary inflammation

\section{Introduction}

Transient Receptor Potential Vanilloid-4 (TRPV4) channels are mechanosensitive calcium channels that have been detected in various lung cell types, including the epithelium and endothelium, ${ }^{1}$ alveolar macrophages, ${ }^{2}$ airway smooth muscle cells ${ }^{3}$ and circulating inflammatory cells, especially neutrophils. ${ }^{4}$ Recently, the TRPV4 calcium channels have received great attention for their role in mediating acute lung injury and pulmonary inflammatory responses. ${ }^{5,6}$ TRPV4 are activated by mechanical stress and have been implicated in the pathogenesis of pulmonary inflammation in in-vivo models of ventilator-induced lung injury (VILI). ${ }^{2}$ Their role, however, is not limited to mechanical stress-induced lung injury, given that blocking TRPV4 channels also prevented acid-induced lung injury in mice. ${ }^{4}$

During exacerbations of airway diseases, such as asthma and COPD, the lung is exposed to significant mechanical stress through increased airway narrowing (bronchoconstriction), hyperinflation and rigorous contractions of the inspiratory muscles (mainly the diaphragm), resulting in large negative swings in intrathoracic pressure. ${ }^{7,8}$ In an effort to mimic the mechanical consequences of severe airway obstruction, our research group has established a mouse model of resistive 
breathing through tracheal banding that induces lung injury and pulmonary inflammation. ${ }^{9}$ An acute bout of inspiratory resistive breathing in rats results in activation of mechanosensitive pathways in the lung by increasing the phosphorylation of Src kinase, a central kinase in mechanotransduction. ${ }^{10}$ However, the role of TRPV4 channels in resistive breathing induced acute lung injury is unknown.

Thus, we hypothesized that the mechanosensitive TRPV4 channels are implicated in the pathogenesis of resistive breathing-induced lung injury and that TRPV4 inhibition protects from the devastating effects of resistive breathing. For this purpose, we have employed the mouse model of resistive breathing through tracheal banding and TRPV4 channel inhibition was studied in both a preventive (prior to stimuli) and a therapeutic manner (after stimuli).

\section{Materials and Methods}

\section{Subjects}

Adult male C57BL/6 mice (8-10 weeks old) were used in this study. Animals were purchased by the Biomedical Sciences Research Center "A. Fleming" and were housed in a 12-hour day/night cycle at the Experimental Surgery unit of Evangelismos Hospital provided food and water ad libitum.

\section{Animal Model of Resistive Breathing Through Tracheal Banding}

An animal model of resistive breathing through tracheal banding was employed, as previously described by our group. ${ }^{9}$ Briefly, mice were anaesthetized with an intraperitoneal injection of ketamine $(90 \mathrm{mg} / \mathrm{kg})$ and xylazine $(5 \mathrm{mg} / \mathrm{kg})$ and placed under a surgical microscope. The trachea was exposed, and a nylon band of a pre-specified length was introduced below trachea and sutured around it, to provoke a $50 \%$ reduction of its surface area. This has been shown before to induce a resistive load of $\mathrm{Ppl} / \mathrm{Ppl}$, max $\sim 0.22$, increased twofold compared to sham controls. ${ }^{11}$ Following recovery from anesthesia, the mice were returned to their cage. Resistive breathing was applied for 24 hours. Sham operated animals were used for comparisons (controls). A 24-hour time frame was chosen to investigate the acute effects of resistive breathing. Based on the time course presented in the supplementary methods, 24 hours is also the time point where a complete spectrum of RBinduced lung injury is firstly observed, in terms of inflammation (Figure S2, S4 and Table S1), lung permeability (Figure S1) and mechanics derangement (igure S3).

\section{TRPV4 Inhibition}

TRPV4 inhibition was performed in both preventive and therapeutic manner. For preventive action two doses of the specific TRPV4 inhibitor, HC-067047 (10 mg/kg ip in 3\% DMSO), were administered 30 min prior to tracheal banding and at 12 hours. To estimate the therapeutical potential of TRPV4 inhibition only one dose of HC-067047 was administered at 12 hours of tracheal banding. Comparisons were performed with mice receiving only the diluents (3\% DMSO in normal saline). This dose of HC-067047 has been previously shown to achieve a drug plasma level above half maximal inhibitory concentration (IC50) in rats for at least 4 hours and has been also used in vivo in mice. ${ }^{12}$

\section{Mechanical Parameters of the Respiratory System}

Following 24 hours of resistive breathing, the animals were anaesthetized with an intraperitoneal injection of ketamine $(90 \mathrm{mg} / \mathrm{kg})$ and xylazine $(10 \mathrm{mg} / \mathrm{kg})$, the sutures and the nylon band around the trachea were carefully removed and the mice were tracheostomized. Then, the mice were connected to a small animal ventilator (FlexiVent, Scireq) to measure respiratory system mechanics. The impedance of the respiratory system was measured by the forced oscillation technique (FOT). Impedance $(Z)$ was then fitted to constant phase model: $\mathrm{Zrs}(\mathrm{f})=\mathrm{Rn}+\mathrm{i} 2 \pi \mathrm{fI}+(\mathrm{G}-\mathrm{iH}) /(2 \pi \mathrm{f})^{\mathrm{a}}$, where $\mathrm{Rn}$ is the newtonian resistance of the airways, $i$ is the imaginary unit, $f$ is the frequency, $I$ is the inertance of the gas in the airways, $\mathrm{G}$ represents tissue damping/viscance (a parameter closely related to tissue resistance that reflects the energy dissipation in the alveoli) and $\mathrm{H}$ represents tissue elasticity. Alpha can be calculated through the equation $\alpha=(2 / \pi) \arctan (\mathrm{H} / \mathrm{G}){ }^{13}$ A static pressure-volume curve was also performed to measure static compliance (slope of the mid linear part of the expiratory P-V curve) and hysteresis, as previously described. ${ }^{14}$ Thirty seconds prior to measurements, the lung volume history was once standardized by performing a deep inspiration to $30 \mathrm{cmH} 20$. 


\section{Bronchoalveolar Lavage Fluid}

Following measurement of the mechanical parameters of the respiratory system, the mice received an additional dose of anaesthesia and were sacrificed by exsanguination (vena cava dissection) after collecting a blood sample. The thoracic cavity was then opened and the left main bronchus was temporarily ligated. Three aliquots of $0.5 \mathrm{~mL}$ normal saline were instilled into the right lung and were gently withdrawn. Bronchoalveolar lavage (BAL) fluid was then centrifuged (300xg, $5 \mathrm{~min}$ ), the supernatant was stored at $-80 \mathrm{C}$ for further analysis and the cell pellet was reconstituted in $1 \mathrm{~mL}$ of normal saline.

\section{Cell Count (Total - Differential)}

Total cell counts were measured with Trypan blue stain and aliquots were pelleted on glass slides by cytocentrifugation. Differential counts were performed on May-Grünwald-stained cytospins and percentages of macrophages, neutrophils, lymphocytes and eosinophils/basophils were determined by counting their number in 300 cells. The number of eosinophils/basophils was negligible and was not further analyzed.

\section{Total Protein and Cytokine Levels in BAL Fluid}

Total protein concentration of BAL fluid was measured, using a colorimetric protein assay according to the manufacturer (BioRad, USA). Bovine serum albumin was used to create standard curves. The protein levels of IL-6, a cytokine that is consistently upregulated is the lung, diaphragm and blood following resistive breathing in both animal and human studies $^{15-17}$ and keratinocyte-chemoattractant (KC), a major neutrophil chemoattractant, were measured with ELISA from BAL fluid supernatant with the standard protocol supplied by the manufacturer (DuoSet ELISA, R\&D Systems, Minneapolis, USA).

\section{Lung Histology}

Following BAL, the right main bronchus was ligated and removed. The left lung was then fixed with $4 \%$ formaldehyde under $20 \mathrm{~cm} \mathrm{H} 2 \mathrm{O}$ pressure and removed. After 24 hours, the lung tissue was embedded in paraffin and haematoxylin and eosin stained lung tissue sections were acquired using conventional techniques. A lung injury score was determined, as previously described, ${ }^{15}$ based on the following histological features: (i) focal alveolar membrane thickening, (ii) capillary congestion, (iii) intra-alveolar haemorrhage, (iv) interstitial, (v) intra-alveolar leukocyte infiltration and (vi) presence of edema. Each feature was scored from 0 to 3 based on its absence (0) or presence to a mild (1), moderate (2), or severe (3) degree.

\section{Surfactant Protein D (SpD) Levels in Blood Samples}

SpD protein level was estimated from a blood sample with ELISA with the standard protocol supplied by the manufacturer (DuoSet ELISA, R\&D Systems, Minneapolis, USA).

\section{Statistical Analysis}

Data presented as mean \pm SD. Statistical analysis was performed with one way ANOVA and the Fisher LSD test for post hoc comparisons. Statistical significance was defined by a $\mathrm{p}$ value $<0.05$.

\section{Results}

\section{Mechanical Parameters of the Respiratory System}

As shown in Figures 1A, 24 hrs of RB resulted in a right- and downward shift of the static pressure-volume curve of the respiratory system, consistent with the presence of lung injury. Static compliance $\left(\mathrm{C}_{\mathrm{st}}\right)$ of the respiratory system was decreased, compared to control $(\mathrm{p}=0.004$, Figure 1B). Both preventive and therapeutic TRPV4 inhibition ameliorated the decrease in $\mathrm{C}_{\mathrm{st}}$ following RB. Interestingly, TRPV4 inhibition resulted also in a marginal increase in $\mathrm{C}_{\mathrm{st}}$ in quietly breathing animals (control), compared to diluent $(3 \%$ DMSO) $(\mathrm{p}=0.049)$. Accordingly, respiratory system mechanics measurement by the forced oscillation technique revealed that $24 \mathrm{~h}$ of RB increased tissue viscance $(\mathrm{G})$ and elasticity $(\mathrm{H})$ 
A

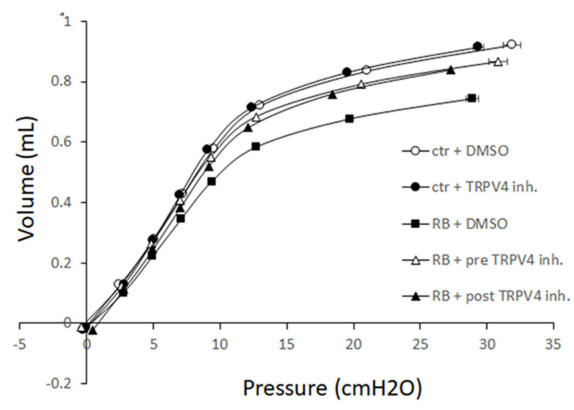

C

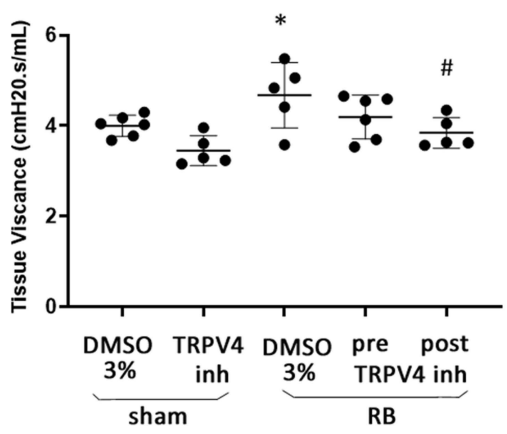

B

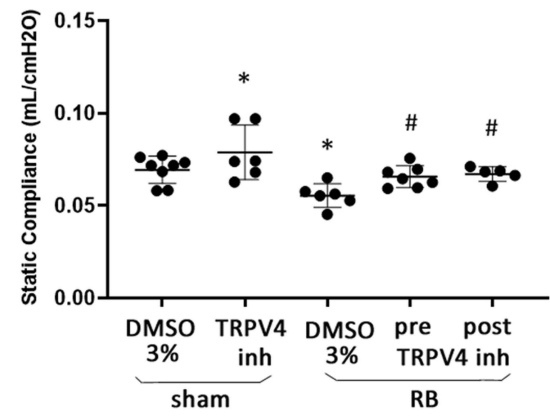

D

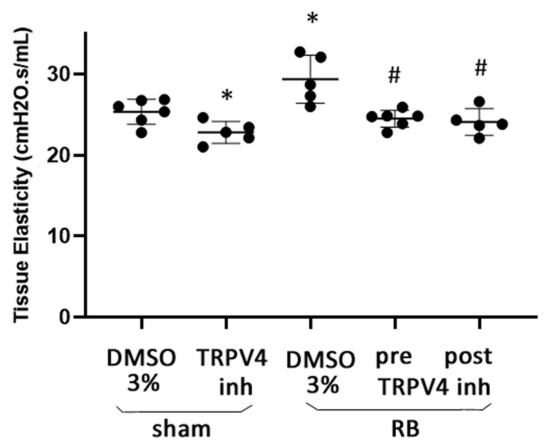

Figure I Mechanical properties of the respiratory system following resistive breathing and the effect of TRPV4 inhibition. (A) RB provokes a right- and downward shift of the static pressure-volume (P-V) curve, consisted with the presence of lung injury. Following TRPV4 inhibition the mean P-V curve moves towards control curves. To minimize figure complexity, only the expiratory part of the mean P-V curve for every experimental group is depicted. (B) 24 hrs of RB decreases static compliance of the respiratory system, an effect that is ameliorated following TRPV4 inhibition. (C) Tissue viscance was increased after $24 \mathrm{hrs}$ of RB, an effect not noticed after TRPV4 inhibition. (D) TRPV4 inhibition ameliorated the increase in tissue elasticity after $24 \mathrm{~h}$ of RB, as measured with FOT. Data presented as mean \pm stdev, $\mathrm{n}=5-8$ per group, ${ }^{*} \mathrm{P}<$ 0.05 to sham $+3 \%$ DMSO, \#p $<0.05$ to $R B+3 \%$ DMSO.

( $\mathrm{G}, \mathrm{p}=0.02$ to $\mathrm{ctr}, \mathrm{H}, \mathrm{p}=0.001$ to $\mathrm{ctr}$ ), an effect that was not observed following either preventive or therapeutic TPV4 inhibition (Figure 1C and D). Newtonian resistance $(\mathrm{Rn})$ was not affected by resistive breathing (ANOVA, $\mathrm{p}=0.45$ ).

\section{Lung Histology}

As previously reported by our group, RB significantly increased the total lung injury score, compared to control ( $\sim 5$-fold, $\mathrm{p}<0.001$, Figure 2). Prophylactic TRPV4 inhibition completely ameliorated this increase, since the lung injury score returned to control values ( $p<0.001$ to RB). Therapeutic TRPV4 inhibition exerted a partial reparative effect, since lung injury was attenuated compared to RB $(p=0.003)$ but remained noticeable compared to control $(p=0.005)$. (For a full description of the changes in individual histological features, see Table 1).

\section{Total and Differential Cell Count in BAL Fluid}

Following $24 \mathrm{~h}$ of $\mathrm{RB}$ a $\sim 3.8$-fold increase of total cell number in BAL fluid was noticed compared to sham controls $(\mathrm{p}<0.001$, Figure 3A), due to an increase in both macrophage and neutrophil count $(\mathrm{p}<0.001$ and $\mathrm{p}<0.001$, respectively). Preventive TRPV4 inhibition completely ameliorated the increase of both macrophages and neutrophils $(p<0.001$ and $p<0.001$ to RB, respectively, Figure 3B and C). Therapeutic TRPV4 inhibition exerted a partial protection, since both macrophages and neutrophils, although reduced compared to $\mathrm{RB}$ ( $\mathrm{p}=0.008$ and $\mathrm{p}<0.001$, respectively), remained elevated compared to sham controls ( $p<0.001$ and $p=0.02$, respectively, Figure $3 B$ and $C$ ). Following $R B$, a significant increase of lymphocyte count was noticed $\left(\mathrm{x} 10^{\wedge} 4 / \mathrm{mL}\right.$, sham $0.017 \pm 0.018$, RB $\left.0.21 \pm 0.16, \mathrm{p}=0.004\right)$, although lymphocytes were the least abundant cell type in BAL fluid $(<1 \%$ of total). Both prophylactic and therapeutic TRPV4 inhibition blocked the increase of lymphocytes following $\mathrm{RB}(\mathrm{p}=0.01$ and $\mathrm{p}=0.03$ to $\mathrm{RB}$, respectively). 
A

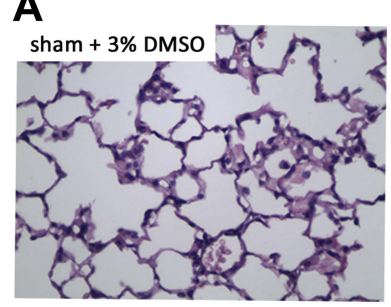

D

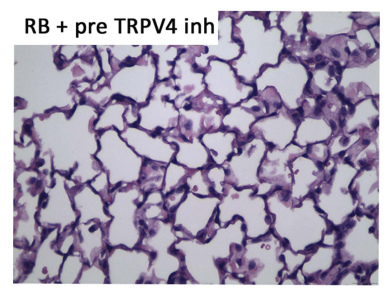

B

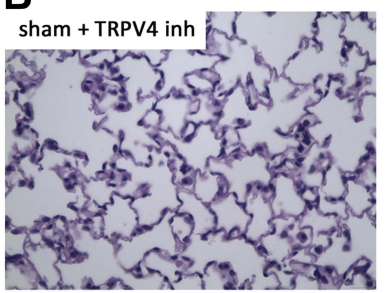

E

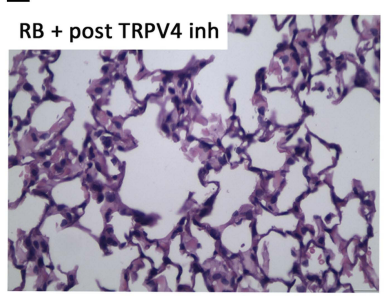

C

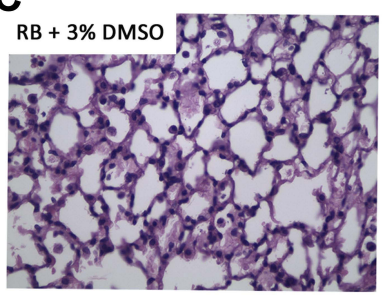

$\mathbf{F}$

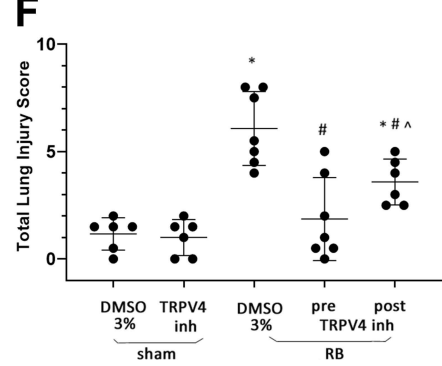

Figure 2 Histological analysis of lung tissue sections following resistive breathing and the effect of TRPV4 inhibition. Representative figures of H\&E stained lung tissue sections (400x magnification) for sham plus 3\% DMSO (A), sham plus TRPV4 inhibitor (B), RB plus 3\% DMSO (C), RB plus prophylactic TRPV4 inhibition (D) and RB plus therapeutic TRPV4 inhibition (E). Note that RB is associated with the presence of increased alveolar membrane focal thickening, capillary congestion, intra-alveolar hemorrhage, interstitial and intra-alveolar leukocyte infiltration and the presence of edema, resulting in an increased total lung injury score (F). Prophylactic TRPV4 inhibition completely ameliorates the aforementioned features, while therapeutic inhibition exerts a partial protective effect. Data presented as mean \pm stdev, $n=6-7$ per group, ${ }^{*} \mathrm{p}<$ 0.05 to sham + 3\% DMSO, \#p $<0.05$ to RB+ 3\% DMSO, ${ }^{\wedge} p<0.05$ to RB+preTRPV4 inh.

\section{Cytokine Levels in BAL Fluid}

$\mathrm{RB}$ resulted in a $\sim 2.8$-fold and $\sim 25$-fold increase of IL-6 and $\mathrm{KC}$ levels in BAL fluid, compared to control ( $\mathrm{p}=0.01$ and $\mathrm{p}<0.001$, respectively, Figure 4A and B). Following both prophylactic and therapeutic TRPV4 inhibition, IL-6 levels returned to control values ( $p=0.0012$ and $p=0.006$ to $R B$, respectively), whereas $\mathrm{KC}$ levels decreased compared to $\mathrm{RB}$, but remained elevated compared to control values (prophylactic inhibition, $p<0.001$ to ctr and RB, therapeutic inhibition, $\mathrm{p}=0.018$ to $\mathrm{ctr}, \mathrm{p}<0.001$ to $\mathrm{RB})$.

\section{Total Protein Levels in BAL Fluid and Surfactant Protein D (SpD) Levels in Blood Samples}

Following $24 \mathrm{hrs}$ of $\mathrm{RB}$, total protein levels in BAL were elevated ( $p=0.01$ to $\mathrm{ctr}$ ) but returned to control values following both preventive and therapeutic TRPV4 inhibition (Figure 5A). Resistive breathing resulted in an increased plasma level of SpD ( $\mathrm{p}<0.001$ to ctr), (Figure 5B). Both protective and therapeutic TRPV4 inhibition significantly decreased SpD plasma level following resistive breathing $[\sim 43 \%(\mathrm{p}<0.001)$ and $\sim 57 \%(\mathrm{p}<0.001)$ reduction, respectively], although $\mathrm{SpD}$ level remained elevated compared to $\operatorname{ctr}(\mathrm{p}<0.001$ and $\mathrm{p}=0.008$, respectively).

Table I Histological Features of Lung Injury Following Resistive Breathing and the Protective Effect of TRPV4 Inhibition

\begin{tabular}{|c|c|c|c|c|c|c|c|}
\hline & $\begin{array}{c}\text { Focal } \\
\text { Thickening }\end{array}$ & $\begin{array}{c}\text { Capillary } \\
\text { Congestion }\end{array}$ & $\begin{array}{c}\text { Intra- } \\
\text { Alveolar } \\
\text { Hemorrhage }\end{array}$ & $\begin{array}{l}\text { Interstitial } \\
\text { Leukocyte } \\
\text { Infiltration }\end{array}$ & $\begin{array}{c}\text { Intra-Alveolar } \\
\text { Leukocyte } \\
\text { Infiltration }\end{array}$ & Edema & Total \\
\hline Sham + 3\% DMSO & $0.41 \pm 0.37$ & $0.16 \pm 0.25$ & $0.08 \pm 0.20$ & $0.33 \pm 0.40$ & $0.08 \pm 0.20$ & $0.08 \pm 0.20$ & $1.16 \pm 0.75$ \\
\hline Sham + trpv4 inh & $0.33 \pm 0.25$ & $0.25 \pm 0.27$ & $0.00 \pm 0.00$ & $0.25 \pm 0.27$ & $0.08 \pm 0.20$ & $0.08 \pm 0.20$ & $1 \pm 0.83$ \\
\hline $\mathrm{RB}+3 \% \mathrm{DMSO}$ & $1.21 \pm 0.0 .39 *$ & $1.07 \pm 0.0 .18 *$ & $1.00 \pm 0.40 *$ & $1.14 \pm 0.37 *$ & $1.07 \pm 0.18 *$ & $0.57 \pm 0.60 *$ & $6.07 \pm 1.7 \mid *$ \\
\hline $\mathrm{RB}+$ pre trpv4 inh & $0.57 \pm 0.53 \#$ & $0.28 \pm 0.39 \#$ & $0.14 \pm 0.37 \#$ & $0.5 \pm 0.40 \#$ & $0.07 \pm 0.18 \#$ & $0.28 \pm 0.39$ & $1.85 \pm 1.93 \#$ \\
\hline $\mathrm{RB}+$ post trpv4 inh & $0.75 \pm 0.27 \#$ & $0.75 \pm 0.27 * \wedge$ & $0.16 \pm 0.40 \#$ & $0.75 \pm 0.27$ & $0.41 \pm 0.37 *^{\wedge} \wedge$ & $0.75 \pm 0.27 * \wedge$ & $3.58 \pm 1.06 * \#^{\wedge}$ \\
\hline
\end{tabular}

Notes: Data presented as mean \pm stdev. ${ }^{*} p<0.05$ to sham $+3 \%$ DMSO, \# $p<0.05$ to RB+ 3\% DMSO, ${ }^{\wedge} p<0.05$ to RB+pre TRPV4 inh. 
A

B
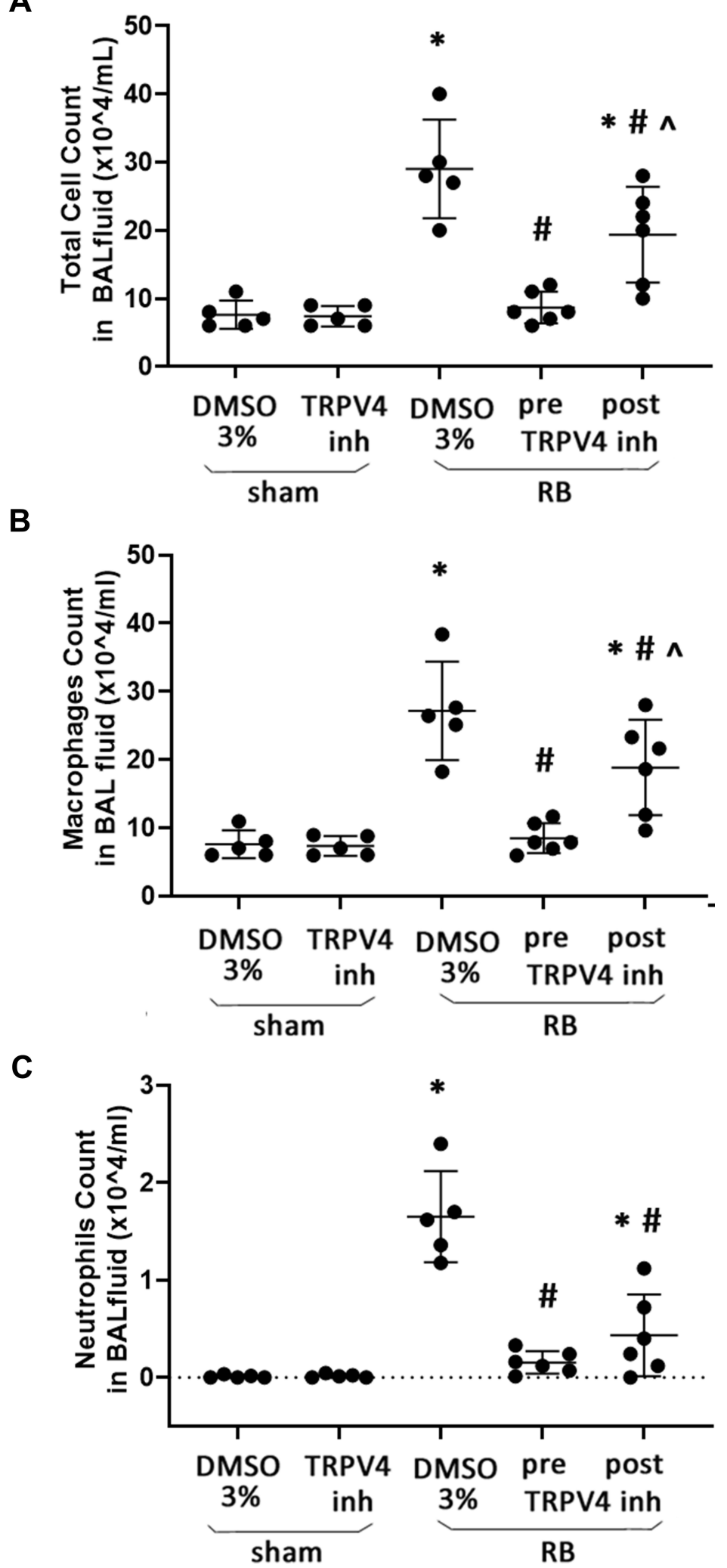

Figure 3 Total and differential cell count in BAL fluid following resistive breathing and the effect of TRPV4 inhibition. 24h or RB was resulted in increased total cell numbers in BAL fluid (A), due to raised macrophages (B) and neutrophil counts (C). Preventive TRPV4 inhibition completely abolished this effect, while therapeutic TRPV4 inhibition exerted a partial protection. Data presented as mean \pm stdev, $n=5-6$ per group, ${ }^{*} p<0.05$ to sham $+3 \%$ DMSO, \#p $<0.05$ to RB $+3 \%$ DMSO, ${ }^{\wedge}<$ 0.05 to RB+preTRPV4 inh. 
A

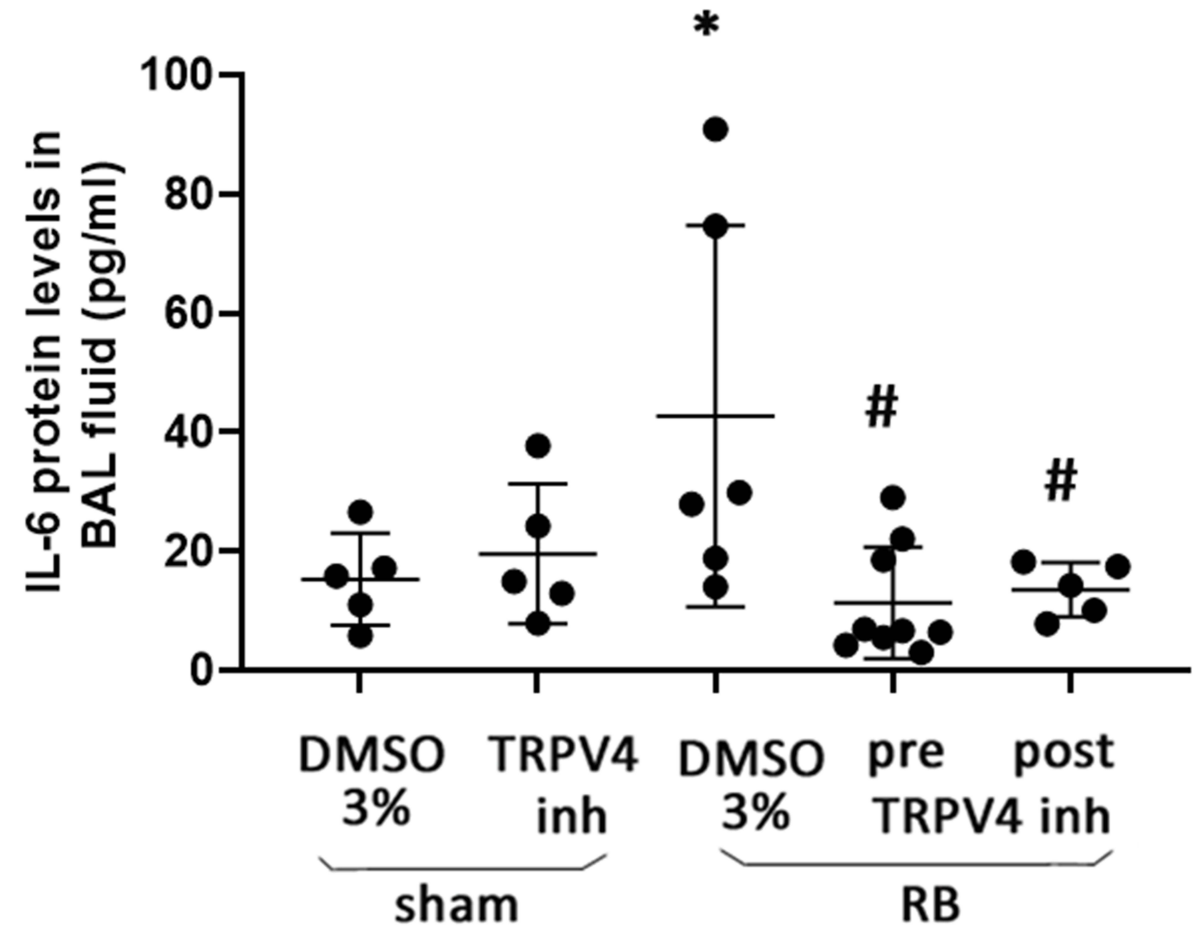

B

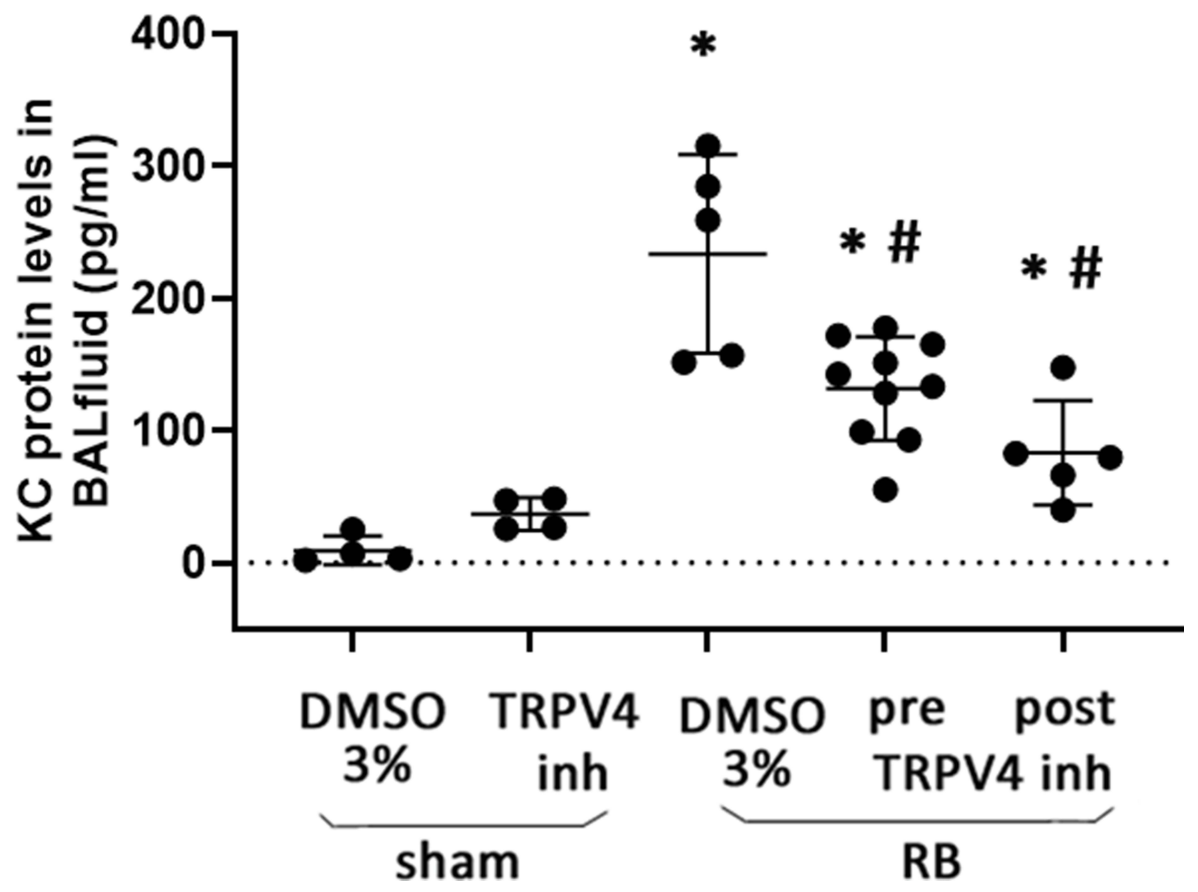

Figure 4 Protein levels of inflammatory cytokines in BAL fluid following resistive breathing and the effect of TRPV4 inhibition. (A) IL-6 protein levels were elevated in the BAL fluid following $24 \mathrm{hrs}$ of RB, an effect that was ameliorated by both prophylactic and therapeutic TRPV4 inhibition. (B) The administration of TRPV4 inhibitor, HC067047, exerted a partial protective effect against the upregulation of KC following 24hrs of RB. Data presented as mean \pm stdev, $n=4-10$ per group, *p $<0.05$ to sham + $3 \%$ DMSO, \#p $<0.05$ to RB+3\% DMSO.

\section{Discussion}

The main finding of our study is that inhibition of TRPV4 channels reduces pulmonary inflammation and lung permeability derangement following resistive breathing, both in a preventive and a therapeutic manner.

As previously described by our group, ${ }^{9}$ resistive breathing through tracheal banding in mice resulted in lung injury, as assessed by the induction of pulmonary inflammation, increase in lung permeability and alterations in respiratory system 

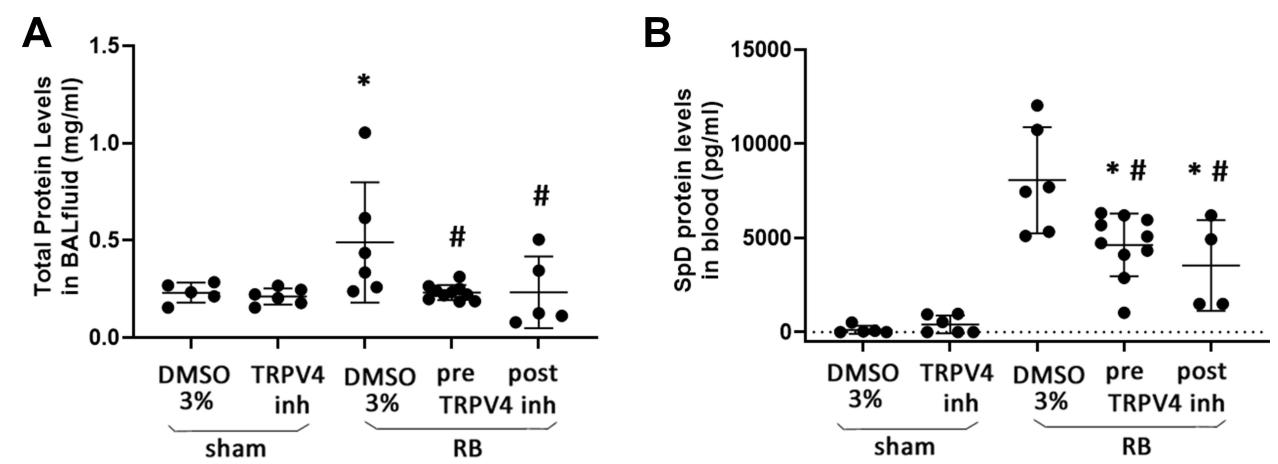

Figure 5 Total protein level in BAL fluid and Surfactant protein D (SpD) plasma levels following resistive breathing and the effect of TRPV4 inhibition. (A) 24hrs of RB increased total protein levels in BAL fluid (an indirect marker of increased lung permeability), an effect that was blocked following both prophylactic and therapeutic TRPV4 inhibition. (B) TRPV4 inhibition partially prevented the increase of SpD plasma levels. Data presented as mean \pm stdev, $n=4-10$ per group, *p $<0.05$ to sham $+3 \%$ DMSO, $\# p<0.05$ to $R B+3 \%$ DMSO.

mechanics, ie, reduction in static compliance. Spontaneous breathing through tracheal banding results in increased mechanical stress in lung, due to increased airway resistance and negative intrathoracic pressures provoked by rigorous contractions of the inspiratory muscles. ${ }^{11}$ Although a direct injurious effect of the increased mechanical forces cannot be excluded, ${ }^{18}$ resistive breathing is found to result in activation of "mechanosensitive" pathways in the lung that at least in part mediate the induction of lung injury, such as the Src kinase pathway. ${ }^{10,19}$ This is in accordance with other forms of mechanical stress-induced lung injury, such as ventilator-induced lung injury (VILI). ${ }^{20}$

Over the last years, TRPV4 channels have been recognized, as a key "mechanosensitive" mediator in the lung. ${ }^{21}$ In vivo, high tidal volume ventilation led to activation of TRPV4 channels in the lung, presumably through phosphorylation. ${ }^{22}$ Interestingly, our group has shown that Src kinase is activated in the lung shortly following resistive breathing ${ }^{10}$ and Src kinase can activate TRPV4 through tyrosine phosphorylation ${ }^{23}$ raising the interesting possibility that TRPV4 channels might have been activated in our model secondary to Src activation. Nevertheless, TRPV4 activation in the lung is multifactorial since TRPV4 can be also chemically activated, such as through a 14.15-epoxyeicosatrienoic (EET) acid-mediated mechanism. ${ }^{1}$ Although the mechanism for the activation of TRPV4 following resistive breathing is not determined and was beyond the scope of our study, the use of a highly specific TRPV4 inhibitor allowed us to investigate the role of TRPV4 in RB-induced lung injury.

According to our results, TRPV4 inhibition dampened the inflammatory response in the lung following resistive breathing. Histological analysis and measurement of total and differential cell count in BAL fluid revealed a reduced influx of leukocytes (both macrophages and neutrophils) in the lung following TRPV4 inhibition, an effect seen especially in the preventive HC-067047 administration. This is in accordance with previous studies showing an antiinflammatory effect of TRPV4 inhibition in acid-induced and ventilator-induced acute lung injury. ${ }^{22,24}$ Moreover, TRPV4 inhibition resulted in a significant reduction in cytokine levels in the BAL fluid, such as KC, which is the mouse analog for IL-8, a major chemokine for neutrophils. Indeed, TRPV4 channels have been shown to play an important role in neutrophil infiltration in the lung and inhibition of TRPV4 channels in circulating neutrophils, ameliorated neutrophil activation. ${ }^{4}$

Cumulative evidence has established a clear role of TRPV4 activation in disruption of the alveolar-capillary membrane integrity. ${ }^{1,5}$ Our findings are in accordance with this role, since TRPV4 inhibition was found to ameliorate the increase of total protein in BAL fluid following resistive breathing, an indirect marker of increased lung permeability. Activation of TRPV4 channels induces an increase in total protein levels in the lung through a metalloproteinase (MMP)-2 and -9 mediated mechanism ${ }^{25}$ and we have shown that an acute session of inspiratory resistive breathing induces MMP-9 expression in the lung. ${ }^{26}$ Interestingly, in a model of hydrostatic pulmonary edema through increased lung microvascular pressure, TRPV4 activation resulted in increased lung permeability, but also activated a negative-feedback loop through induction of cGMP pathway, suggesting that cGMP inactivates TRPV4 function in lung microvascular endothelial cells. ${ }^{27}$ Our group has shown that resistive breathing downregulates the cGMP pathway and that restoration of cGMP levels restores lung epithelial permeability following resistive breathing, ${ }^{9}$ suggesting that the described downregulation of cGMP pathway in our model could be 
contributing to a continuing activation of TRPV4 channels. In support of the role of TRPV4 in the resistive breathing induced lung injury, TRPV4 inhibition, both prior and after initiation of resistive breathing caused a significant decrease in blood SpD levels, a potential serum biomarker of lung epithelial injury, ${ }^{28}$ which is correlated with clinical outcomes in COPD patients (the prototype disease of resistive breathing). ${ }^{29}$ It should be mentioned that although $\mathrm{SpD}$ increase in plasma does reflect epithelial injury that injury could be a result of epithelial interaction with leukocytes rather than the direct consequence of epithelial TRPV4 activation through mechanotransduction. ${ }^{5}$ TRPV4 has been shown to influence neutrophil recruitment in the lung, the expression of chemotactic cytokines and ROS production by neutrophils ${ }^{4}$ and the expression of adhesion molecules. ${ }^{30}$ Indeed, in our experiment, as stated above, TRPV4 inhibition prevented the leukocyte increase in the BAL fluid and decreased KC expression. In contrast, however, in a mouse model of acid-induced lung injury, the TRPV4-mediated increase in lung permeability was attributed to TRPV4 expressed in the lung rather than to TRPV4 expressed in circulating neutrophils. ${ }^{4}$

To our knowledge, this is the first study to evaluate TRPV4 role in pulmonary inflammation in an animal model of severe airway obstruction. Our results may have clinical implications for diseases of severe airway obstruction, such as post obstructive pulmonary edema or exacerbations of airway diseases, such as COPD and asthma. As previously reported by our group, the magnitude of resistive load and the time frame used in our animal model were chosen to mimic the mechanical consequences observed in exacerbations of obstructive pulmonary diseases. ${ }^{9,15,19}$ Indeed, there are human clinical data on the consequences of COPD exacerbations that are consistent with our findings, mainly from imaging studies, ${ }^{31}$ since obtaining histological samples or performing lung permeability studies during acute exacerbation of COPD is challenging. In an autopsy study where in 6 patients death was attributed to respiratory failure secondary to COPD exacerbation per se, 3 of these patients were exhibiting bilateral infiltrates and 2 vascular congestion on the chest x-ray on Hospital admission. ${ }^{32}$ Moreover, William NP et al, 2018, have reported pulmonary infiltrates in $20.1 \%$ of COPD exacerbation that correlate with more intense systematic inflammation. ${ }^{33}$

Thus, our results raise the intriguing possibility of a therapeutic role of TRPV4 inhibition for diseases of airway obstruction, especially during exacerbations. TRPV4 expression has been detected not only in the lung periphery (alveolar epithelial and microvascular endothelial cells), but also in cultured human airway smooth muscle cells ${ }^{34}$ and airway sensory neurons ${ }^{35}$ suggesting a potentially important functional role of TRPV4 in the lung. In vitro, TRPV4 activation results in airway constriction in isolated human bronchus sections, ${ }^{3}$ suggesting that inhibition might be having bronchodilatory effects. TRPV4 knockout mice exhibit reduced lung inflammation following exposure to cigarette smoke, ${ }^{36}$ suggesting that TRPV4 inhibition might have antiiflammatory effects, as documented in our current study. Interestingly, TRPV4 polymorphisms have been associated with COPD in humans. ${ }^{37}$

An important finding of our study is that TRPV4 inhibition retained its anti-inflammatory action, even when performed 12 hours after the initiation of resistive breathing. This increases the clinical implications of TRPV4 inhibition as a potential therapeutic intervention after injury has occurred. In fact, this possibility has been previously also suggested in models of chemically induced acute lung injury. ${ }^{24}$ Further studies are needed to fully elucidate the therapeutic potential of TRPV4 manipulation with possible distinction between sterile (as in our model) or infectious inflammation. ${ }^{38}$ This is also applicable to diseases of airway obstruction, where the phenotyping between infectious vs non-infectious causes of exacerbations is currently challenging. Thus, together with the recently described roles of TRPV4 channels in control of breathing by inducing rapid shallow breathing ${ }^{39}$ and as mediators of hypoxic pulmonary vasoconstriction, ${ }^{40}$ TRPV4 channels is an attractive though complex/multifactorial therapeutic target.

\section{Conclusions}

In conclusion, our study provide evidence for a protective effect of TRPV4 inhibition both in a preventive and therapeutic manner in an animal model of severe airway obstruction.

\section{Abbreviations}

BAL, bronchoalveolar lavage; COPD, chronic obstructive pulmonary disease; DMSO, dimethyl sulfoxide; RB, resistive breathing; SpD, surfactant protein D; TRPV4, transient receptor potential vanilloid-4. 


\section{Data Sharing Statement}

The data used in the current study are available from the corresponding author on reasonable request.

\section{Ethics Approval}

All procedures, described in Materials and Methods, were approved by the ethical committee of the experimental surgery Department of Evangelismos Hospital and are in accordance with the national law that follows the European Union Directive (2010/63/EU) on the protection of animals used for scientific purposes.

\section{Acknowledgments}

The authors would like to thank Dr. Dionysios Tsoukalas for his valuable contribution in the revision process of the manuscript.

\section{Author Contributions}

All authors made substantial contributions to conception and design, acquisition of data, or analysis and interpretation of data; took part in drafting the article or revising it critically for important intellectual content; gave final approval of the version to be published; and agree to be accountable for all aspects of the work.

\section{Funding}

This work was supported by a Grant from the Hellenic Thoracic Society.

\section{Disclosure}

The authors have no conflicts of interest to disclose.

\section{References}

1. Alvarez DF, King JA, Weber D, Addison E, Liedtke W, Townsley MI. Transient receptor potential vanilloid 4-mediated disruption of the alveolar septal barrier: a novel mechanism of acute lung injury. Circ Res. 2006;99(9):988-995. doi:10.1161/01.RES.0000247065.11756.19

2. Hamanaka K, Jian MY, Townsley MI, et al. TRPV4 channels augment macrophage activation and ventilator-induced lung injury. Am $J$ Physiol Lung Cell Mol Physiol. 2010;299(3):L353-362. doi:10.1152/ajplung.00315.2009

3. McAlexander MA, Luttmann MA, Hunsberger GE, Undem BJ. Transient receptor potential vanilloid 4 activation constricts the human bronchus via the release of cysteinyl leukotrienes. J Pharmacol Exp Ther. 2014;349(1):118-125. doi:10.1124/jpet.113.210203

4. Yin J, Michalick L, Tang C, et al. Role of Transient Receptor Potential Vanilloid 4 in Neutrophil Activation and Acute Lung Injury. Am J Respir Cell Mol Biol. 2016;54(3):370-383. doi:10.1165/rcmb.2014-0225OC

5. Morty RE, Kuebler WM. TRPV4: an exciting new target to promote alveolocapillary barrier function. Am J Physiol Lung Cell Mol Physiol. 2014;307(11):L817-821. doi:10.1152/ajplung.00254.2014

6. Scheraga RG, Southern BD, Grove LM, Olman MA. The Role of Transient Receptor Potential Vanilloid 4 in Pulmonary Inflammatory Diseases. Front Immunol. 2017;8:503. doi:10.3389/fimmu.2017.00503

7. O’Donnell DE, Parker CM. COPD exacerbations. 3: pathophysiology. Thorax. 2006;61(4):354-361. doi:10.1136/thx.2005.041830

8. Veerati PC, Mitchel JA, Reid AT, et al. Airway mechanical compression: its role in asthma pathogenesis and progression. Eur Respir Rev. 2020;29:157. doi:10.1183/16000617.0123-2019

9. Glynos C, Toumpanakis D, Loverdos K, et al. Guanylyl cyclase activation reverses resistive breathing-induced lung injury and inflammation. $A m$ J Respir Cell Mol Biol. 2015;52(6):762-771. doi:10.1165/rcmb.2014-0092OC

10. Toumpanakis D, Vassilakopoulou V, Sigala I, et al. The role of Src \& ERK1/2 kinases in inspiratory resistive breathing induced acute lung injury and inflammation. Respir Res. 2017;18(1):209. doi:10.1186/s12931-017-0694-7

11. Toumpanakis D, Mizi E, Vassilakopoulou V, et al. Spontaneous Breathing Through Increased Airway Resistance Augments Elastase-Induced Pulmonary Emphysema. Int J Chron Obstruct Pulmon Dis. 2020;15:1679-1688. doi:10.2147/COPD.S256750

12. Everaerts W, Zhen X, Ghosh D, et al. Inhibition of the cation channel TRPV4 improves bladder function in mice and rats with cyclophosphamide-induced cystitis. Proc Natl Acad Sci U S A. 2010;107(44):19084-19089. doi:10.1073/pnas.1005333107

13. Bates JH, Irvin CG. Measuring lung function in mice: the phenotyping uncertainty principle. J Appl Physiol. 2003;94(4):1297-1306. doi:10.1152/ japplphysiol.00706.2002

14. Kastis GA, Toumpanakis D, Loverdos K, et al. Dose- and time-dependent effects of lipopolysaccharide on technetium-99-m-labeled diethylene-triamine pentaacetatic acid clearance, respiratory system mechanics and pulmonary inflammation. Exp Biol Med (Maywood). 2013;238(2):209-222. doi:10.1258/ebm.2012.012313

15. Toumpanakis D, Kastis GA, Zacharatos P, et al. Inspiratory resistive breathing induces acute lung injury. Am J Respir Crit Care Med. $2010 ; 182$ (9):1129-1136. doi:10.1164/rccm.201001-0116OC

16. Vassilakopoulos T, Divangahi M, Rallis G, et al. Differential cytokine gene expression in the diaphragm in response to strenuous resistive breathing. Am J Respir Crit Care Med. 2004;170(2):154-161. doi:10.1164/rccm.200308-10710C 
17. Vassilakopoulos T, Katsaounou P, Karatza MH, Kollintza A, Zakynthinos S, Roussos C. Strenuous resistive breathing induces plasma cytokines: role of antioxidants and monocytes. Am J Respir Crit Care Med. 2002;166(12 Pt 1):1572-1578. doi:10.1164/rccm.200203-177OC

18. West JB. Invited review: pulmonary capillary stress failure. J Appl Physiol. 2000;89(6):2483-2489. doi:10.1152/jappl.2000.89.6.2483

19. Vassilakopoulos T, Toumpanakis D. Can resistive breathing injure the lung? Implications for COPD exacerbations. Int J Chron Obstruct Pulmon Dis. 2016;11:2377-2384. doi:10.2147/COPD.S113877

20. Curley GF, Laffey JG, Zhang H, Slutsky AS. Biotrauma and Ventilator-Induced Lung Injury: clinical Implications. Chest. 2016 ;150(5):1109-1117. doi:10.1016/j.chest.2016.07.019

21. Parker JC, Townsley MI. Control of TRPV4 and Its Effect on the Lung. In: Kamkin A, Kiseleva I, editors. Mechanosensitivity and Mechanotransduction. Springer; 2011:239-254.

22. Michalick L, Erfinanda L, Weichelt U, van der Giet M, Liedtke W, Kuebler WM. Transient Receptor Potential Vanilloid 4 and Serum Glucocorticoid-regulated Kinase 1 Are Critical Mediators of Lung Injury in Overventilated Mice In Vivo. Anesthesiology. 2017 ;126(2):300-311. doi:10.1097/ALN.0000000000001443

23. Xu H, Zhao H, Tian W, Yoshida K, Roullet JB, Cohen DM. Regulation of a transient receptor potential (TRP) channel by tyrosine phosphorylation. SRC family kinase-dependent tyrosine phosphorylation of TRPV4 on TYR-253 mediates its response to hypotonic stress. $J$ Biol Chem. 2003;278 (13):11520-11527. doi:10.1074/jbc.M211061200

24. Balakrishna S, Song W, Achanta S, et al. TRPV4 inhibition counteracts edema and inflammation and improves pulmonary function and oxygen saturation in chemically induced acute lung injury. Am J Physiol Lung Cell Mol Physiol. 2014;307(2):L158-172. doi:10.1152/ajplung.00065.2014

25. Villalta PC, Rocic P, Townsley MI. Role of MMP2 and MMP9 in TRPV4-induced lung injury. Am J Physiol Lung Cell Mol Physiol. 2014;307(8): L652-659. doi:10.1152/ajplung.00113.2014

26. Toumpanakis D, Noussia O, Sigala I, et al. Inspiratory resistive breathing induces MMP-9 and MMP-12 expression in the lung. Am J Physiol Lung Cell Mol Physiol. 2015;308(7):L683-692. doi:10.1152/ajplung.00133.2014

27. Yin J, Hoffmann J, Kaestle SM, et al. Negative-feedback loop attenuates hydrostatic lung edema via a cGMP-dependent regulation of transient receptor potential vanilloid 4. Circ Res. 2008;102(8):966-974. doi:10.1161/CIRCRESAHA.107.168724

28. Eisner MD, Parsons P, Matthay MA, Ware L, Greene K. Acute Respiratory Distress Syndrome N. Plasma surfactant protein levels and clinical outcomes in patients with acute lung injury. Thorax. 2003;58(11):983-988. doi:10.1136/thorax.58.11.983

29. Sin DD, Leung R, Gan WQ, Man SP. Circulating surfactant protein D as a potential lung-specific biomarker of health outcomes in COPD: a pilot study. BMC Pulm Med. 2007;7:13. doi:10.1186/1471-2466-7-13

30. Wu S, Jian MY, Xu YC, et al. Ca2+ entry via alpha1G and TRPV4 channels differentially regulates surface expression of P-selectin and barrier integrity in pulmonary capillary endothelium. Am J Physiol Lung Cell Mol Physiol. 2009;297(4):L650-657. doi:10.1152/ajplung.00015.2009

31. Rangelov BA, Young AL, Jacob J, et al. Thoracic Imaging at Exacerbation of Chronic Obstructive Pulmonary Disease: a Systematic Review. Int J Chron Obstruct Pulmon Dis. 2020;15:1751-1787. doi:10.2147/COPD.S250746

32. Zvezdin B, Milutinov S, Kojicic M, et al. A postmortem analysis of major causes of early death in patients hospitalized with COPD exacerbation. Chest. 2009;136(2):376-380. doi:10.1378/chest.08-2918

33. Williams NP, Ostridge K, Devaster JM, et al. Impact of radiologically stratified exacerbations: insights into pneumonia aetiology in COPD. Respir Res. 2018;19(1):143. doi:10.1186/s12931-018-0842-8

34. Jia Y, Wang X, Varty L, et al. Functional TRPV4 channels are expressed in human airway smooth muscle cells. Am J Physiol Lung Cell Mol Physiol. 2004;287(2):L272-278. doi:10.1152/ajplung.00393.2003

35. Bonvini SJ, Birrell MA, Grace MS, et al. Transient receptor potential cation channel, subfamily V, member 4 and airway sensory afferent activation: role of adenosine triphosphate. J Allergy Clin Immunol. 2016;138(1):249-261 e212. doi:10.1016/j.jaci.2015.10.044

36. Baxter M, Eltom S, Dekkak B, et al. Role of transient receptor potential and pannexin channels in cigarette smoke-triggered ATP release in the lung. Thorax. 2014;69(12):1080-1089. doi:10.1136/thoraxjnl-2014-205467

37. Zhu G, Investigators I, Gulsvik A, et al. Association of TRPV4 gene polymorphisms with chronic obstructive pulmonary disease. Hum Mol Genet. 2009;18(11):2053-2062. doi:10.1093/hmg/ddp111

38. Michalick L, Kuebler WM. TRPV4-A Missing Link Between Mechanosensation and Immunity. Front Immunol. 2020;11:413. doi:10.3389/ fimmu.2020.00413

39. Gu QD, Moss CR 2nd, Kettelhut KL, Gilbert CA, Hu H. Activation of TRPV4 Regulates Respiration through Indirect Activation of Bronchopulmonary Sensory Neurons. Front Physiol. 2016;7:65. doi:10.3389/fphys.2016.00065

40. Goldenberg NM, Wang L, Ranke H, Liedtke W, Tabuchi A, Kuebler WM. TRPV4 Is Required for Hypoxic Pulmonary Vasoconstriction. Anesthesiology. 2015;122(6):1338-1348. doi:10.1097/ALN.0000000000000647

International Journal of Chronic Obstructive Pulmonary Disease

Dovepress

\section{Publish your work in this journal}

The International Journal of COPD is an international, peer-reviewed journal of therapeutics and pharmacology focusing on concise rapid reporting of clinical studies and reviews in COPD. Special focus is given to the pathophysiological processes underlying the disease, intervention programs, patient focused education, and self management protocols. This journal is indexed on PubMed Central, MedLine and CAS. The manuscript management system is completely online and includes a very quick and fair peer-review system, which is all easy to use. Visit http://www. dovepress.com/testimonials.php to read real quotes from published authors.

Submit your manuscript here: https://www.dovepress.com/international-journal-of-chronic-obstructive-pulmonary-disease-journal 